\title{
LA CONCEPTUALIZACIÓN DE LOS EVENTOS DE CAMBIO DE ESTADO Y LA ALTERNANCIA LÁBIL EN ESPAÑOL*
}

\section{THE CONCEPTUALIZATION OF CHANGE OF STATE EVENTS AND THE LABILE ALTERNATION IN SPANISH}

\author{
Margot Vivanco \\ Universidad Complutense de Madrid \\ margotvivanco@gmail.com
}

Recibido: 02/09/2017

Aceptado: 10/10/2017

\begin{abstract}
Resumen
Las teorías sobre la conceptualización de los eventos de cambio de estado (cf. Haspelmath 1993) han tenido una enorme presencia en los estudios sobre la alternancia causativo-inacusativa a partir del trabajo de Levin y Rappaport Hovav (1995), tanto en relación con el problema de la marcación de la alternancia como con el problema de qué verbos participan de ella. Este artículo reflexiona sobre su utilidad teórica y defiende la necesidad de redefinirlas mediante la aplicación del Principio de la Correspondencia FormaFrecuencia (Haspelmath et al. 2014; Heidinger 2015), gracias al cual se evitan planteamientos circulares. Nuestro objeto de estudio serán los escasos verbos que participan de la alternancia lábil en español, cuya resistencia a la expansión de se como marca regular de la alternancia es una cuestión idiosincrásica que obedece a la interacción de
\end{abstract}

\begin{abstract}
The theories about the conceptualization of change of state events (cf. Haspelmath 1993) have had an enormous influence on the studies about the causative-unaccusative alternation since Levin \& Rappaport Hovav's work (1995), both with regard to the morphological marking problem and the problem of which verbs may undergo the alternation. This paper reflects on their theoretical usefulness and defends the need of refining them by applying the Form-Frequency Correspondence Principle (Haspelmath et al. 2014; Heidinger 2015), which avoids circular statements. Our case in point are the few verbs undergoing the labile alternation in Spanish, whose resistance against the expansion of se as a regular marker is an idiosyncratic matter determined by the interaction of both cognitive factors,
\end{abstract}

\footnotetext{
* Este estudio ha sido realizado en el marco del grupo de investigación Gramática Teórica del Español (930590) de la Universidad Complutense de Madrid. Quiero agradecerles sus comentarios y aportaciones a Cristina Sánchez López, a Antonio Fábregas y a los evaluadores anónimos de la revista, pues han contribuido enormemente a la mejora de este trabajo.
}

Para citar este artículo / To cite this article: Vivanco, Margot (2017). La conceptualización de los eventos de cambio de estado y la alternancia lábil en español. ELUA, 31: 327-347. doi: 10.14198/ ELUA2017.31.17

Enlace / Link: http://dx.doi.org/10.14198/ELUA2017.31.17 
factores cognitivos, como la conceptualización de los eventos, y de factores gramaticales, como el aspecto y la estructura escalar.

PALABRAS CLAVE: conceptualización de los eventos; alternancia anticausativa; alternancia lábil. such as the conceptualization of events, and grammatical factors, such as aspect and scale structure.

KEYWORDS: conceptualization of events; anticausative alternation; labile alternation.

\section{INTRODUCCIÓN}

Los estudios sobre eventos de cambio de estado y la alternancia causativo-inacusativa a menudo emplean escalas de espontaneidad para ordenar los verbos y explicar ciertas diferencias entre ellos. Dichas escalas se basan en la idea de que los verbos que denotan eventos conceptualizados por los hablantes como altamente susceptibles de ocurrir de manera espontánea tienden a ser empleados más frecuentemente como intransitivos, sin marca morfológica alguna; en cambio, aquellos verbos que denotan eventos conceptualizados como poco susceptibles de ocurrir espontáneamente tienden a ser empleados como transitivos y a marcar la variante intransitiva, por ser la menos esperable (cf. Centineo 1995; Schäfer 2008; Letuchyi 2010; Heidinger 2014, 2015; Alexiadou 2014). Esta hipótesis tiene su origen en estudios tipológicos (Nedjalkov 1969; Haspelmath 1993) que tratan de explicar el grado de complejidad formal -qué variante es la morfológicamente marcada en la alternancia- en función del grado de complejidad conceptual. Posteriormente, estas escalas han sido utilizadas para explicar tres de los grandes problemas relacionados con los verbos de cambio: i) la capacidad para participar de la alternancia -los verbos que denotan eventos más espontáneos tienden a no alternar-, ii) la direccionalidad de la alternancia -los verbos más espontáneos podrían ser en realidad básicamente intransitivos, sujetos a un proceso de causativización-y iii) la marcación de la alternancia -la variante más esperable de los verbos más espontáneos es la intransitiva y, por lo tanto, esta suele ser la menos marcada morfológicamente.

Si bien las fuertes tendencias que evidencian los estudios tipológicos han de ser tenidas en cuenta por cualquier estudio sobre la alternancia, resulta difícil determinar y, sobre todo, formalizar cuál es exactamente el rol desempeñado por la conceptualización de los hablantes, pues se trata de una cuestión idiosincrásica, no sistemática.

Este trabajo realiza una revisión crítica del uso teórico que se ha dado a la hipótesis de la conceptualización en los estudios sobre la alternancia, concretamente en relación al problema de qué verbos pueden participar de ella y al problema de la marcación. Así mismo, comprobaremos hasta qué punto dicha hipótesis resulta útil para comprender el fenómeno de la alternancia lábil en español, que afecta tan solo a una veintena de verbos y parece, a primera vista, una irregularidad del sistema.

Se argumentará que la hipótesis de la conceptualización tiene un importante valor a nivel descriptivo pero es peligrosa a nivel explicativo, por su carácter circular: se deduce el grado de espontaneidad de un verbo en función de su marcación, la cual a su vez está supuestamente determinada por su grado de espontaneidad. Para romper con la circularidad es necesario recurrir al Principio de la Correspondencia Forma-Frecuencia (Haspelmath et al. 2014), asumiendo que la manera en que los hablantes conceptualizan un evento queda reflejada en el número de usos causativos e inacusativos que hacen de un verbo. Observaremos que, efectivamente, los verbos más usados como inacusativos tienden a ser los menos marcados; sin embargo, dado que estas 
hipótesis plantean tendencias pero no reglas, se subrayará la importancia de considerar otros factores gramaticales en relación con los problemas planteados. Una peculiaridad de algunos verbos lábiles del español es que carecen de una dimensión escalar, a diferencia del resto de verbos de cambio de estado, lo cual remite a las diferencias aspectuales, determinadas por la estructura escalar, que se han encontrado entre estos verbos y los anticausativos (cf. Vivanco 2016).

\section{BREVE PANORAMA TIPOLÓGICO DE LA ALTERNANCIA. LOS VERBOS LÁBILES DEL ESPAÑOL: ¿RARA AVIS?}

La alternancia causativo-inacusativa se expresa mediante diversos mecanismos morfosintácticos en las lenguas del mundo. Haspelmath (1993), siguiendo a Nedjalkov (1969) y a Nedjalkov y Silnitsky (1973), elabora un estudio tipológico en el cual distingue tres grandes tipos de alternancia: la causativa, la anticausativa, y la no direccional.

En la alternancia causativa, la variante inacusativa es la más simple morfológicamente, mientras que la transitiva es la que se deriva por medio de un sufijo o de un verbo auxiliar ${ }^{1}$ (1); en cambio, en la alternancia anticausativa ${ }^{2}$, la variante marcada es la inacusativa (2):
(1) Duy-s intr. $_{\text {a }} \rightarrow$ a-duy-ebs tr. $_{\text {. }}$
(2) a. Katat'-sja $\mathbf{a}_{\text {intr. }} \leftarrow$ katat' $_{\text {tr. }}$
b. Khul-naa intr. $_{\text {khol-naa }}$
c. Romper-se intr. $_{\text {r. }} \leftarrow$ romper $_{\text {tr. }}$

(Georgiano: ‘cocinar')

(Ruso: 'rodar')

(Hindi-Urdu: 'abrir')

En las alternancias no direccionales, donde ninguna de las dos formas verbales deriva morfológicamente de la otra, se pueden distinguir tres subtipos. En primer lugar, la alternancia equipolente es aquella en la que tanto la variante causativa como la inacusativa comparten una misma raíz, a la que se agregan morfemas distintos (3). En segundo lugar, en la alternancia supletiva se utilizan dos raíces verbales diferentes para expresar la oposición causativo / inacusativo (4). Si bien todas las lenguas cuentan con pares de este tipo, no es el patrón dominante en ninguna. Finalmente, la alternancia lábil es aquella en la que la misma forma verbal se emplea para los dos significados, sin que se produzca alteración morfológica alguna (5).

(3) Atum-aru $\mathbf{i n t r}_{\text {int }} \leftrightarrow$ atum-eru

(Japonés: 'reunir')

(4) a. Goret' / žeč',

b. Morir / matar; entrar / meter; salir / sacar.

(Ruso: 'arder' / 'quemar')

(5) a. Break $_{\text {intr. }} /$ break $_{\text {tr. }}$ b. Cambiar ${ }_{\text {intr. }} /$ cambiar $_{\text {tr. }}$

(Inglés: 'romper')

La alternancia lábil afecta tan solo a una veintena de verbos en español, como adelgazar, aumentar, blanquear, cambiar, disminuir, empeorar, encanecer, engordar, enfermar, enloquecer, enmudecer, envejecer, hervir o resucitar, mientras que la alternancia anticausativa, expresada

1 Los ejemplos a continuación son de Haspelmath (1993: 91-92), salvo los del español.

2 El término fue acuñado por Nedjalkov y Silnitsky (1973), pero hoy en día muchos trabajos lo emplean para referir a la alternancia causativo-inacusativa en general. 
mediante el clítico $s e^{3}(2 \mathrm{c})$, es el mecanismo empleado por la inmensa mayoría de los verbos alternantes, como romper(se), secar(se), enfriar(se), enfadar(se), cerrar(se), pudrir(se), etc.

Existe además en español un grupo de verbos -caramelizar, cicatrizar, cristalizar, cuajar, despertar, encoger, enmohecer, ennegrecer, enrojecer, mejorar, rejuvenecer, reventar- que toman el se en la variante inacusativa de manera opcional (6), es decir, que participan indistintamente de la alternancia anticausativa y de la lábil, incrementando levemente el número de verbos con los que este último mecanismo está disponible 4 . Siguiendo la terminología de Schäfer (2008), nos referiremos a los verbos que participan de la alternancia anticausativa como "clase A", a los que participan de la lábil como "clase B" y a los que participan de ambas como "clase C". La clase C y, en consecuencia, la B, están sujetas a una importante variación dialectal e incluso idiolectal": si bien para todos los hablantes de español existen estos grupos, no todos consideran que estén conformados por los mismos verbos.

(6) a. El humo ennegreció la pared.

b. La pared (se) ennegreció.

De acuerdo con Haspelmath (1993), la alternancia anticausativa es el mecanismo más empleado en las lenguas del mundo, seguido, en este orden, por la alternancia causativa, la equipolente, la lábil y la supletiva. Las lenguas que, como el inglés, utilizan exclusivamente la alternancia lábil son escasas -se trata de un recurso típico de las lenguas ergativo-absolutivas, como las lenguas del Dagestán ${ }^{6}-$; de hecho, es frecuente que este mecanismo compita con otro u otros en las lenguas indo-europeas. De este modo, encontramos la alternancia lábil junto a la anticausativa en las lenguas romances, en las eslavas, en alemán, en griego, etc., si bien la distribución de algunos verbos concretos varía -así, el verbo despertar pertenece a la clase $\mathrm{C}$ en español, pero a la A en francés e italiano.

La alternancia lábil se documenta desde los primeros textos latinos, así como en las lenguas indo-europeas antiguas (cf. Gianollo 2014). En el caso del griego clásico, parece ser que la alternancia lábil fue desarrollándose a partir del uso causativo ocasional de verbos que eran originalmente inacusativos no alternantes (7-8), y finalmente llegó a reemplazar a la alternancia anticausativa como mecanismo regular (cf. Lavidas 2009; Karantzola y Lavidas 2014).

(KyL 2014: 1030)

(Griego clásico)

(7) hóti oukh hekō’n examartánō all' amathíai têi emêi que no a propósito errar.PRES.ACT.1sG. pero ignorancia.DAT.SG. art.DAT.SG. 1SG.DAT. 'Que no yerro a propósito, sino por ignorancia'.

(KyL 2014: 1031)

(8) kaì exếmartes tòn laón mou tòn Israêl

(Griego romano-helenístico)

y errar.AOR.ACT.2SG. art.ACU.SG. gente.ACU.SG. 1SG.GEN. artc.ACU.SG. Israel

'Y tú hiciste errar a mi pueblo, Israel.'

3 Esto sucede en la mayoría de las lenguas indoeuropeas, como el italiano, francés, rumano, alemán, ruso, sueco, noruego, ruso, checo, búlgaro, etc.

4 Esto sucede también en otras muchas lenguas, como por ejemplo el francés, el italiano, el alemán o el griego moderno.

5 También puede haber variación entre hablantes de distintas generaciones.

6 Lenguas caucásicas nororientales habladas en ciertas regiones de Rusia y Azerbaiyán. 
Hoy la clase B es más numerosa que la A en griego moderno; en cambio, en las lenguas romances la situación es generalmente la contraria. Después de haber atravesado periodos de expansión en la historia del propio latín, la alternancia lábil ha experimentado un declive más o menos rápido y más o menos intenso en las distintas lenguas romances, que han elegido unánimemente se como la expresión formal de la alternancia causativo-inacusativa. Así, para Heidinger (2014), los verbos de la clase B del francés son, en cierto sentido, reliquias históricas dentro del sistema. Concretamente, este autor habla de "persistencia" diacrónica desde el latín, frente a la expansión de la forma competidora se como marca regular de la alternancia.

En francés, el número de verbos lábiles oscila entre los 300 (Rothenberg 1974) y los 500, lo cual contrasta significativamente con el español, donde se estima que no hay más de 20 ó 40 verbos de este tipo ${ }^{7}$. A primera vista, esta cifra hace pensar que en español la alternancia lábil es, efectivamente, una irregularidad en el sistema y que la clase B es un residuo histórico, un reducto conformado por unos pocos verbos fosilizados. Sin embargo, tal afirmación requiere el apoyo de una investigación diacrónica que excede los propósitos de este trabajo.

Por otra parte, la clase B no solo tiene el problema de ser pequeña en español, sino también el de ser extremadamente heterogénea. En ella encontramos verbos derivados de adjetivos de escala abierta -con comportamiento aspectual variable, como adelgazar y envejecer-, verbos derivados de adjetivos de escala cerrada de dos puntos - que dan lugar a logros, como enfermar y enloquecer- y otros logros no deadjetivales como hervir, cambiar y resucitar ${ }^{8}$. No encontramos, sin embargo, verbos derivados de escalas cerradas de múltiples puntos que den lugar a realizaciones: estos se encuentran exclusivamente en la clase A.

Por estas razones, los numerosos intentos por encontrar una única propiedad común que explique la persistencia diacrónica o la identidad sincrónica de las formaciones lábiles frente a las formaciones regulares con se han encontrado serias dificultades, enfrentándose a constantes excepciones y comportamientos idiosincrásicos que impiden formular una generalización plenamente satisfactoria.

La convivencia en una misma lengua de dos -o más- mecanismos para expresar la alternancia causativo-inacusativa es bastante frecuente pero plantea, no obstante, un dilema fundamental que ha recibido una considerable atención en la bibliografía (cf. Labelle 1990, 1992; Centineo 1995; Folli 2001; Alexiadou y Anagnostopoulou 2004; Schäfer 2008, Labelle y Doron 2010; Kailuweit 2012; Heidinger 2015): ¿existen diferencias semánticas y/o sintácticas entre las variantes lábiles y las anticausativas que expliquen la elección entre una y otra, justificando así su coexistencia?

La bibliografía ofrece dos grandes familias de hipótesis - no mutuamente excluyentes y a menudo aplicadas conjuntamente (Labelle y Doron 2010; Heidinger 2014, 2015)- que tratan de solucionar este problema: la hipótesis de la conceptualización de los eventos, sobre la que versa este artículo, y la hipótesis del contraste aspectual.

Podemos englobar bajo la etiqueta "hipótesis del contraste aspectual" una serie de propuestas que han señalado ciertas diferencias aspectuales entre las anticausativas marcadas y no marcadas, especialmente en las lenguas romances (Labelle 1990, 1992; Folli 2001; Labelle y Doron 2010; Cennamo y Jezek 2011; Kailuweit 2011; Cennamo 2012), pero también en griego moderno (Alexiadou y Anagnostopoulou 2004), latín e islandés antiguo (Cennamo et

7 Dependiendo de la variedad dialectal e idiolectal.

8 A estos verbos se suman los de la clase C, también escasos y también heterogéneos -deadjetivales de comportamiento aspectual variable como ennegrecer y logros como despertar. 
al. 2015) ${ }^{9}$. De acuerdo con esta hipótesis, la expresión morfológica del cambio en la diátesis es sensible a las propiedades aspectuales del evento, pues los verbos de comportamiento aspectual variable de la clase $\mathrm{C}$ solo admiten lecturas atélicas en ausencia de se:

(9) El paciente $(*$ se) mejoró durante una semana.

De acuerdo con Cennamo et al. (2015), en latín el contraste era robusto: la alternancia anticausativa era un mecanismo reservado a los verbos inherentemente télicos, y la lábil se aplicaba a los de comportamiento aspectual variable. No obstante, se fue extendiéndose a todo tipo de verbos ya en latín, de manera que, a pesar de que se siguen apreciando contrastes como el de (9), hoy encontramos verbos télicos y atélicos en las clases A, B y C, como comentábamos anteriormente.

Vivanco (2016) reformula la hipótesis del aspecto argumentando que este viene en realidad determinado por la estructura escalar lexicalizada por los verbos de cambio de estado. Aunque por razones de espacio no podemos profundizar en esta cuestión aquí, cabe señalar que, de acuerdo con Vivanco (2016), las realizaciones pertenecen exclusivamente a la clase A en español, y que los logros de cambio pueden ser simples (no escalares) o complejos (escalas de dos puntos) en función de la presencia o ausencia de se.

Esta hipótesis da cuenta de todos los verbos de la clase B en español -que consta de verbos de escala abierta y logros simples-, pero no explica por sí sola que haya otros similares (calentar, verbo atélico de escala abierta) en la A. Nótese que el verdadero problema no es la expansión de se a verbos como calentar como marca por defecto de la alternancia, sino su "no expansión" a los veinte o cuarenta verbos de la clase B. Para aclarar esto, resulta necesario recurrir a otros factores de índole idiosincrásica, como la conceptualización de los eventos.

El siguiente apartado profundiza sobre los planteamientos, consecuencias y problemas teóricos de la hipótesis de la conceptualización, observando la utilidad concreta que tiene respecto a los verbos lábiles.

\section{LAS TEORÍAS SOBRE LA CONCEPTUALIZACIÓN DE LOS EVENTOS}

La hipótesis de la conceptualización se articula fundamentalmente en torno a los siguientes dos principios:

(10) The form-meaning complexity isomorphism principle: (Haspelmath, 2014: 590) More complex meanings are expressed by more complex forms; i.e. by more coding material.

(11) Meta-iconic markedness principle:

(Givón 1991: 106) Categories that are cognitively marked tend also to be structurally marked.

De acuerdo con estos principios, las expresiones más esperables y/o con significados menos complejos ${ }^{10}$ se expresan mediante formas lingüísticas más simples. Los hablantes, de manera subjetiva, tienden a concebir los eventos como más o menos propensos a suceder

9 Remito al lector a Schäfer (2008) y Vivanco (2016) para una revisión crítica de estas propuestas.

10 Lo relevante a este respecto no es el significado objetivo, sino el conceptual (Haspelmath 1993). 
espontáneamente -sin la intervención de un agente o causa-; así, los verbos que denotan eventos conceptualizados como altamente espontáneos tienden a construirse de manera natural como inacusativos.

\begin{tabular}{|c|c|c|c|c|}
\hline & $\begin{array}{c}\text { Alternancia } \\
\text { anticausativa }\end{array}$ & $\begin{array}{c}\text { Alternancia } \\
\text { causativa }\end{array}$ & $\begin{array}{c}\text { Alternancia } \\
\text { lábil }\end{array}$ & $\begin{array}{c}\text { Alternancia } \\
\text { equipolente }\end{array}$ \\
\hline Hervir & 0,5 & 11,5 & 6 & 3 \\
\hline Llenar & 8 & 5 & 3 & 5 \\
\hline Cerrar & 15,5 & 1 & 2 & 2,5 \\
\hline
\end{tabular}

Tabla 1. Verbos y tipos de alternancia (adaptada de Haspelmath 1993: 104).

La Tabla 1 recoge, a título ilustrativo, 3 de los 31 verbos estudiados por Haspelmath; las cifras corresponden al número de lenguas en las que se da cada tipo de alternancia, entre las 21 estudiadas. En ella vemos que hervir presenta una fuerte tendencia a marcar la variante causativa como la menos "esperable desde el punto de vista cognitivo", al contrario que cerrar, que tiende, en la mayoría de las lenguas, a marcar la variante inacusativa. Estos verbos representan los extremos de la escala, pero entre ellos hay otros, como llenar, cuya marcación es menos uniforme a nivel tipológico, lo cual evidencia un mayor grado de indeterminación respecto a la manera en que los hablantes del mundo conceptualizan el evento. Haspelmath et al. (2014: 589) ponen como ejemplo el verbo despertar, que emplea la alternancia causativa en lituano, la anticausativa en francés, la equipolente en alemán y la lábil en griego, y que en español pertenece a la clase C.

En la misma línea, Schäfer (2008) propone una escala de espontaneidad que determina tanto la marcación como la capacidad de un verbo para alternar:

(Adaptado de Schäfer, 2008: 161)

(12) - espontaneidad. ...+espontaneidad

Verbos agentivos

$\varnothing$ Verbos inacusativos

Asesinar

romperse

cambiar ... florecer

Las lenguas difieren respecto al punto de la escala a partir del cual comienzan a emplear morfología extra para "marcar lo inesperado", y también respecto a la manera de conceptualizar ciertos eventos (Schäfer 2008: 161), lo cual explica que las clases A y B no sean uniformes a través de las lenguas y diverjan en número. En la zona media de la escala, entre los verbos como romper(se) y los verbos como cambiar, puede haber un grupo de verbos opcionalmente marcados -los de la clase $\mathrm{C}$-, dentro de un área de indeterminación en la conceptualización.

Volviendo a los verbos lábiles del español, observamos que todos ellos encajan a primera vista con la descripción de "muy propensos a ocurrir de manera espontánea", destacando el subgrupo conformado por adelgazar, engordar, envejecer, resucitar, enfermar, enloquecer y enmudecer que denotan procesos fisiológicos internos ${ }^{11}$. Sin embargo, no hay que olvidar que otros verbos que podríamos considerar igualmente espontáneos se acogen al patrón regular de la clase A, como es el caso de pudrir(se).

11 Malkiel (1941:457) ya señala que ciertos verbos que expresan "phisical grow and decay", como encalvecer, engordar, envejecer o adelgazar alternan sin se. Véase también Bosque (1990). 
El principal problema de la hipótesis de la conceptualización es que puede resultar circular: partiendo de que un verbo como hervir suele formar inacusativas no marcadas en las lenguas del mundo, al contrario que uno como abrir, se concluye que el primero denota un evento concebido como más espontáneo que el segundo y esto, a su vez, es lo que explica la marcación. Así, el propio concepto de "evento espontáneo" resulta difuso: todas las variantes inacusativas de la alternancia denotan eventos espontáneos de cambio frente a las causativas, de modo que tratar de ordenar los eventos en una escala de espontaneidad deja demasiado lugar a la subjetividad.

La intuición parece acertada atendiendo a los extremos de la escala, pero esta no da lugar a un patrón sistemático dentro de una misma lengua -pudrirse frente a hervir-, ni tampoco a nivel tipológico - despertar - o diacrónico -el griego frente al latín. La conceptualización de los eventos por parte de los hablantes es una cuestión idiosincrásica e impredecible que influye de manera significativa, pero no determinante, en el grado de transparencia morfosintáctica que estos eligen para codificar lingüísticamente dichos eventos.

En el apartado 3.2. esta hipótesis será completada con el Principio de la Correspondencia Forma-Frecuencia, que relaciona la frecuencia de usos causativos e inacusativos con la marcación y la conceptualización. Esto paliará en cierta medida el carácter subjetivo de las escalas de espontaneidad: esperamos que los eventos que los hablantes conceptualizan como más espontáneos sean aquellos que los hablantes emplean menos frecuentemente en su variante causativa. Antes de ello, sin embargo, vamos a deternernos a revisar el papel que la hipótesis de la conceptualización ha tenido en relación al problema de determinar qué verbos son los que pueden participar de la alternancia.

\subsection{La conceptualización y qué verbos alternan}

Un problema recurrente en los estudios sobre la alternancia causativo-inacusativa es que, si bien todos los verbos alternantes son de cambio de estado, no todos los verbos de cambio de estado alternan (cf. Chierchia 1989; Levin y Rappaport Hovav 1995; Reinhart 2000; Alexiadou et al. 2006; Horvath y Siloni 2008): ciertos verbos causativos, como asesinar, carecen de variante intransitiva y ciertos inacusativos, como florecer, nunca transitivizan.

En la bibliografía encontramos esencialmente tres maneras de abordar esta cuestión. La primera de ellas asume que el hecho de que un mecanismo esté disponible en una lengua no implica que tenga que aplicarse obligatoriamente en todos los casos posibles (cf. Arad 2005). La segunda considera que las formas aparentemente ausentes existen, de hecho, en algún nivel de representación, aunque no pasen a formar parte del vocabulario (cf. Reinhart 2002; Horvath y Siloni 2008; Fadlon 2012). Finalmente, la tercera vía consiste en buscar factores semánticos adicionales, como la conceptualización de los eventos, que expliquen la (in)capacidad de un verbo para alternar (cf. Levin y Rappaport Hovav 1995 -en adelante LyR-; Alexiadou et al. 2006, 2015).

La hipótesis de conceptualización de los eventos subyace a la gran mayoría de estudios sobre la alternancia desde LyR (1995). El problema de qué verbos aternan y el de la marcación se han tratado tanto conjuntamente como por separado, pero en ambos casos la noción de "espontaneidad" -también "grados de espontaneidad"- ha resultado crucial. Por ello, consideramos necesario dedicar este apartado al debate sobre la dicotomía causa interna / externa que, si bien no es central para el estudio de los verbos lábiles del español, sí lo es para comprender la trascendencia de la teoría de la conceptualización, así como sus riesgos y contradicciones. 
El estudio de Haspelmath (1993) muestra que los verbos conceptualizados como más espontáneos suelen ser no alternantes, como tampoco lo son los que denotan eventos conceptualizados como agentivos. LyR (1995) parten de este trabajo para formular su conocida teoría que divide los verbos alternantes en "externa e internamente causados"12. Desde esta perspectiva, el factor fundamental para diferenciar un verbo de causa interna de uno de causa externa es su participación de la alternancia anticausativa, lo cual deriva en una estipulación circular: los verbos que alternan lo hacen por ser externamente causados, y sabemos que son externamente causados porque alternan.

A nuestro juicio, lo que sucede, más bien, es que todas las construcciones inacusativas denotan eventos que podrían ser descritos como "internamente causados" - espontáneos- y que, en este sentido, se oponen a las construcciones causativas, que son las que verdaderamente denotan eventos "externamente causados". Es decir, la alternancia causativo-inacusativa se produce precisamente cuando un verbo puede denotar indistintamente un evento de cambio de estado interna o externamente causado.

Haspelmath et al. (2014) advierten del peligro de propuestas como la de LyR, en las que la dicotomía causa interna / externa se formaliza como información codificada en las raíces, subrayando que las tendencias tipológicas que ellos describen suelen ser de poca ayuda para el micro estudio de una lengua particular y que la conceptualización subjetiva de los hablantes no debe confundirse con las propiedades léxicas intrínsecas del verbo: lo segundo es estable, pero lo primero, no.

En el modelo de Alexiadou et al. (2006) y Schäfer (2008) también se hace uso de la causación interna / externa para determinar qué verbos alternan. Estos autores, siguiendo a Harley y Noyer (2000), clasifican raíces atendiendo a su significado enciclopédico:

(13) a. Agentivas: no denotan necesariamente eventos de cambio de estado (bailar), pero si lo hacen tienen un componente agentivo que les impide alternar (asesinar, cortar).

b. Internamente causadas: solo pueden denotar eventos espontáneos de cambio (florecer, crecer), luego no alternan.

c. Externamente causadas: solo denotan eventos de cambio desencadenados por una causa externa (destruir, matar), luego no alternan.

d. No especificadas respecto a la causa (cause unspecified): no determinan si el evento de cambio es espontáneo o causado, por lo que alternan (romper, cambiar).

Esta clasificación establece que solo los verbos que pueden denotar eventos interna o externamente causados indistintamente pueden, de hecho, participar de la alternancia. No obstante, como señala Schäfer (2008: 146), la propuesta corre el riesgo de caer en la circularidad en tanto que las distinciones léxicas del tipo 'causa no especificada' y 'causa externa' pueden resultar ser meras etiquetas descriptivas; en este sentido, el intento de explicar el comportamiento sintáctico de los verbos a partir de la información enciclopédica de las raíces puede derivar en una clasificación de las raíces basada en comportamiento sintáctico que exhiben a posteriori. Esta es una de las razones por las que recientemente Alexiadou et al. (2015) han abandonado la clasificación de (17) $)^{13}$.

12 Nótese que LyR establecen, además, una relación entre la causación externa / interna y la dirección de la alternancia: los verbos externamente causados son diádicos $-\mathrm{y}$ sufren un proceso de intransitivización-, mientras que los internamente causados son monádicos en el léxico.

13 Señalan además otras cuestiones por las que la dicotomía causa interna / externa no debe formalizarse en 
Finalmente, los trabajos de McKoon y Macfarland (2000) y Wright (2001, 2002), quienes han demostrado mediante estudios de corpus que ciertos verbos internamente causados pueden, de hecho, alternar (14-15) $)^{14}$, constituyen el argumento empírico que ha motivado el abandono de la dicotomía causa interna / externa por parte tanto de Alexiadou et al. (2015) y Alexiadou (2014) como de Rappaport Hovav y Levin (2012) y Rappaport Hovav (2014).

(14) Salt air and other common pollutants can decay prints.

(Wright 2002: 341)

salado aire y otros comunes contaminantes pueden corromper impresos

'El aire salado y otros contaminantes comunes pueden corromper los impresos.'

(15) Bright sun wilted the roses.

(Wright 2001: 112-115)

brillante sol marchitó las rosas.

'El sol brillante marchitó las rosas.'

Jiménez Fernández y Tubino Blanco (2014) tratan precisamente este tipo de usos causativos en el dialecto andaluz:

(16) Juan entró las sillas en la casa.

(FJyTB, 2014: 8)

Estos autores señalan que si el verbo inacusativo es compatible con el llamado 'se aspectual', sus posibilidades de causativizar aumentan. Así, por ejemplo, entrar es compatible con este se en andaluz - Juan se entró en casa-, pero no en español estándar ${ }^{15}$. En consecuencia, creen que la causativización tiene que ver con las propiedades aspectuales del evento. También apuntan otras dos cuestiones interesantes. La primera de ellas es que los verbos inacusativos de aparición y existencia nunca causativizan, solo los de cambio de estado. La segunda es que en andaluz (17a) significa lo mismo que (17b); es decir, que el causante codificado como directo en (17b) se interpreta en realidad como un causante indirecto. Más adelante veremos que sucede lo mismo con algunos verbos lábiles que, si bien participan de la alternancia, lo hacen de manera infrecuente, como se observa en (18), donde la acción de fumar acelera indirectamente el envejecimiento de la piel, pero no lo causa directamente, en tanto que se trata de un proceso natural que tiene lugar también en las personas no fumadoras:

(17) a. A María se le cayó el colacao.

b. María cayó el colacao.

(18) Fumar te está envejeciendo la piel.

las raíces. En primer lugar, en el marco de la Morfología Distribuida no es posible atribuir a la raíz acategorial información que solo es relevante si se combina con un núcleo verbalizador. En segundo lugar, en ciertos casos, la alternancia está restringida a ciertas combinaciones <verbo + argumento interno> $(*$ La promesa se rompió), lo cual indica que la capacidad para alternar no está determinada léxicamente.

14 Los verbos decay ('corromper', 'pudrir') y wilt ('marchitar') se consideran no alternantes en inglés.

15 Nótese que el verbo entrar participa de la alternancia supletiva en español estándar, y que la variante causativa meter participa a su vez de la alternancia anticausativa: Se me ha metido algo en el ojo. De este modo, entrar y meterse compiten como variantes inacusativas de meter. 
Rappaport Hovav (2014: 21) concurre con Alexiadou et al. (2006) en que los verbos alternantes son precisamente aquellos que pueden expresar eventos interna o externamente causados y va más allá al concluir que esta dicotomía no está codificada en el léxico (contra LyR 1995): “[...] I take the notion of external causation to be a property of event descriptions, and not construals of events determined necessarily by the verb". De manera similar, Alexiadou et al. (2015: 56) llegan a la conclusión de que "the availability of the causative alternation is determined by the conceptualization of events as these are mapped onto vP structures; i.e. v + object combinations".

Haspelmath (1993) y Haspelmath et al. (2014) subrayan que la correspondencia entre la conceptualización y la marcación es tan solo una tendencia, y que esta última puede venir determinada por otros factores adicionales. Uno de ellos es la evolución diacrónica; por ejemplo, los pocos equipolentes del alemán vienen del proto-germánico: aufwachen / aufwecken ('despertar').

No obstante, la variante más simple conceptualmente no siempre es la más antigua, y tampoco es siempre la menos marcada. Por ejemplo, en latín la alternancia lábil convive desde el principio con la forma marcada en $-r$-morfología medio-pasiva ${ }^{16}$. Feltenius (1997) habla de ella como un proceso de intransitivización en aquellos casos en los que la variante causativa es la "original" (la más antigua y/o más frecuente), si bien existe un grupo de verbos que siempre se han documentado como alternantes y que no permiten, por tanto, identificar la variante "original” (cf. Gianollo, 2014).

En definitiva, los verbos de cambio de estado pueden describir indistintamente eventos llevados a cabo por un agente o una fuerza externa o eventos espontáneos, alternando su estructura argumental.

Los inacusativos no alternantes son piezas especializadas en el significado de cambio espontáneo, lo cual no impide, sin embargo, que alternen potencialmente. Algunos cuentan con variantes supletivas - morir / matar-, otros aparecen usados como causativos en ejemplos marginales (16) y todos pueden formar predicados causativos analíticos -Andrea hizo caer a Diana-.

La dicotomía causa interna / externa, en su formulación original, ha quedado obsoleta como herramienta para formalizar la teoría de la conceptualización de los eventos y para determinar la capacidad de los verbos para alternar. De acuerdo con Haspelmath et al. (2014), Rappaport Hovav y Levin (2012) y Alexiadou et al. (2015), las raíces no contienen ese tipo de información, es decir, los verbos no están léxicamente especificados como interna o externamente causados y tampoco están especificados respecto a su participación de la alternancia. Por el contrario, es el predicado $-<$ verbo + argumento interno $>-$ el que tiene en última instancia la capacidad para combinarse o no con un argumento externo.

La hipótesis de la conceptualización de los eventos, en sus formulaciones originales, recoge y describe tendencias tipológicas de gran importancia; no obstante, los intentos de formalizar estas teorías y extraer reglas sistemáticas de ellas han fracasado por incurrir en planteamientos circulares.

16 El mecanismo regular de la alternancia en latín era la morfología medio-pasiva $-r$, que luego se vio reemplaza por el avance de la alternancia lábil y de la anticausativa con se. 


\subsection{La correspondencia forma-frecuencia}

En los apartados anteriores hemos argumentado que la hipótesis de la conceptualización responde de manera muy intuitiva a una serie de tendencias universales, pero deriva en razonamientos teóricos problemáticos tanto si se aplica al problema de la marcación como si se aplica al problema de qué verbos alternan. En este apartado dicha hipótesis será complementada por el Princpio de la Correspondencia Gramatical Forma-Frecuencia (cf. Greenberg 1966; Croft 2003; Bybee 2006; Haspelmath 2008; Haspelmath et al. 2014) en (20), que es la versión sintáctica del Principio de la Correspondencia Forma-Frecuencia" en (19) (Zipf 1935), aplicado generalmente a nivel morfológico.

(19) The form-frequency correspondence principle:

(Haspelmath 2013: 589) Languages tend to use less coding material for more frequent expressions.

(20) The gramatical form-frequency correspondence principle:

(Haspelmath 2013: 590) When two Grammatical patterns that differ minimally in meaning (i.e. patterns that form a semantic opposition) occur with significantly different frequencies, the less frequent pattern tends to be overtly coded (or coded with more coding material), while the more frequent pattern tends to be zero-coded (or coded with less coding material).

Este principio también se basa en la predictibilidad: las expresiones más frecuentes son más predecibles y por lo tanto no necesitan un alto grado de explicitud morfológica. En consecuencia, si un verbo se emplea más habitualmente como causativo, marcará su variante intransitiva por ser la menos esperable y, viceversa, si un verbo se emplea más frecuentemente como intransitivo, tenderá a formar causativas marcadas. Si podemos medir la frecuencia de uso, la circularidad de los planteamientos anteriores se quiebra: si un verbo se usa poco como causativo, obtenemos un dato empírico que apunta a que los hablantes lo conceptualizan efectivamente como "más espontáneo", y es entonces cuando podemos observar si existe además una correlación con la marcación a fin de corroborar que esta se ve afectada, en mayor o menor medida, por la manera en que los hablantes conceptualizan los eventos y utilizan los verbos.

Siguiendo a Heidinger (2015), aplicamos a continuación esta hipótesis a la clase B para comprobar si, como cabría esperar, sus escasos componentes tienen usos causativos infrecuentes.

Heidinger denomina "causalidad" (causalness) al porcentaje de usos causativos de un verbo y realiza un estudio comparativo de corpus basado en 20 verbos del francés y el español en el que observa una fuerte tendencia de aquellos con un alto grado de "causalidad" a formar anticausativas marcadas. En la siguiente tabla hemos continuado y ampliado su trabajo añadiendo varios verbos de las clases A, B y C (con asterisco) a los que ya quedaron recogidos en su estudio. Para ello se ha seguido la misma metodología empleada por este autor: el "grado de causalidad" de un verbo se calcula multiplicando por 100 el número de usos causativos y dividiendo el resultado entre el número total de ejemplos encontrados en CREA $^{17}$-usos causativos e inacusativos-. El número de total de ejemplos manejados en cada caso es similar, en torno a 150 .

17 Mismo corpus de referencia empleado por Heidinger (2015). Los ejemplos se limitan al español de España. 


\begin{tabular}{|c|c|c|}
\hline & Clase & Porcentaje de usos causativos \\
\hline Envejecer & $\mathrm{B}$ & $6{ }^{\prime} 81 \%$ \\
\hline Enfermar* & $\mathrm{B}$ & 7’92\% \\
\hline Cambiar* & $\mathrm{B}$ & $10^{\prime} 68 \%$ \\
\hline Pudrir* & $\mathrm{A}$ & $12^{\prime} 96 \%$ \\
\hline Encanecer* & $\mathrm{B}$ & $15,15 \%$ \\
\hline Hervir & $\mathrm{B}$ & $15,19 \%$ \\
\hline Enmudecer* & $\mathrm{B}$ & $15^{\prime} 42 \%$ \\
\hline Enrojecer & $\mathrm{C}$ & $19,83 \%$ \\
\hline Amarillear & $\mathrm{B}$ & $20 ’ 83$ \\
\hline Cicatrizar* & $\mathrm{C}$ & $30 ’ 37 \%$ \\
\hline Enfriar* & $\mathrm{A}$ & $30{ }^{\prime} 47 \%$ \\
\hline Engordar & $\mathrm{B}$ & $300^{\prime} 61 \%$ \\
\hline Empeorar* & $\mathrm{B}$ & $31 ' 25 \%$ \\
\hline Sanar* & $\mathrm{B}$ & $355^{\prime} 41 \%$ \\
\hline Ablandar & $\mathrm{A}$ & $35{ }^{\prime} 94 \%$ \\
\hline Endurecer & $\mathrm{A}$ & $37^{\prime} 68 \%$ \\
\hline Disminuir & $\mathrm{B}$ & $37^{\prime} 78 \%$ \\
\hline Enloquecer* & $\mathrm{B}$ & $38 ' 13 \%$ \\
\hline Adelgazar & $\mathrm{B}$ & $39 ’ 39 \%$ \\
\hline Intensificar & $\mathrm{A}$ & $39 ’ 39 \%$ \\
\hline Resucitar* & $\mathrm{B}$ & $399^{\prime} 80 \%$ \\
\hline Ensanchar & $\mathrm{A}$ & $40 ’ 30 \%$ \\
\hline Blanquear* & $\mathrm{B}$ & $41 ' 30 \%$ \\
\hline Multiplicar & $\mathrm{A}$ & $42{ }^{\prime} 45 \%$ \\
\hline Mejorar & $\mathrm{C}$ & $44^{\prime} 05 \%$ \\
\hline Aumentar & $\mathrm{B}$ & $44^{\prime} 65 \%$ \\
\hline Rejuvenecer* & $\mathrm{C}$ & $44^{\prime} 83 \%$ \\
\hline Ennegrecer* & $\mathrm{C}$ & $45,33 \%$ \\
\hline Congelar & $\mathrm{A}$ & $48 ' 39 \%$ \\
\hline Agrandar & $\mathrm{A}$ & $49 ’ 09 \%$ \\
\hline Alargar* & $\mathrm{A}$ & $57^{\prime} 40 \%$ \\
\hline Entristecer* & $\mathrm{A}$ & $64{ }^{\prime} 42 \%$ \\
\hline Abrir & $\mathrm{A}$ & $71^{\prime} 50 \%$ \\
\hline Calentar* & $\mathrm{A}$ & $71{ }^{\prime} 87 \%$ \\
\hline Romper* & $\mathrm{A}$ & $81^{\prime} 47 \%$ \\
\hline Cerrar & $\mathrm{A}$ & $84^{\prime} 69 \%$ \\
\hline Mojar & $\mathrm{A}$ & $85^{\prime} 42 \%$ \\
\hline
\end{tabular}

* \# El cáculo del grado de causalidad de blanquear se ha realizado sin tener en cuenta su uso en la colocación blanquear dinero. Es sabido que algunos verbos no alternan en combinación con ciertos argumentos -como el caso de Antonio rompió su promesa / *su promesa se rompió (cf. Levin y Rappaport Hovav 1995)-.\#

Tabla 2. Porcentaje de usos causativos de los verbos alternantes. 
La tabla muestra que a partir de un $40 \%$ de usos causativos hay una fuerte tendencia a utilizar se, que se vuelve sistemática entorno al 50\%. En cambio, la tendencia con menos de un $30 \%$ de usos causativos es no emplear se. La franja entre el $30 \%$ y el $45 \%$ es la zona de indeterminación, donde la alternancia anticausativa y la lábil compiten, con predilección por esta última. Es decir, de acuerdo con la tabla existe una zona exclusiva para la alternancia anticausativa -por encima del $50 \%$ - pero no para la lábil, aunque este patrón sea el dominante por debajo del $40 \%$.

Estos datos evidencian tendencias acusadas pero no dan lugar a reglas sistemáticas pues, siendo el mecanismo por defecto, se se extiende a verbos con muy bajo grado de causalidad -como pudrir (13\%) - al tiempo que otros verbos con grados significativamente altos se mantienen en la clase B -como aumentar (44'65\%). Aún así, observamos que la predicción se cumple y que los verbos que forman inacusativas no marcadas tienden en su mayoría a usarse poco frecuentemente como causativos, lo cual apunta a que los hablantes conceptualizan de manera idiosincrásica los distintos eventos de cambio de estado y a que esto influe en cómo los codifican lingüísticamente.

La conclusión de Heidinger a partir de la comparación entre el francés y el español es similar a la de Schäfer (2008) en relación con su escala de espontaneidad: las dos lenguas difieren respecto al grado de causalidad requerido para comenzar a emplear se; el límite del español es más bajo -entorno al 40\%, frente al 50\% del francés-, lo cual explica que el número de verbos lábiles del español sea mucho menor.

Como quedó expuesto en el apartado anterior, varios autores han planteado que los verbos más espontáneos son los inacusativos no alternantes, pero en realidad a estos verbos no les está completamente vetada la posibilidad de alternar ya que, de hecho, lo hacen de manera marginal o en dialectos no estándar -entrar las sillas-además de contar en ocasiones con variantes supletivas - entrar / meter-y de participar en contrucciones analíticas -hacer florecer-. Los datos de la Tabla 2, así como la variación dialectal e idiolectal en las clases $\mathrm{B}$ y $\mathrm{C}$, sugieren que algunos de los verbos lábiles - por ejemplo, envejecer, con un grado de causalidad del 6' $81 \%$ - podrían ser considerados como inacusativos no alternantes por un cierto número de hablantes.

De hecho, en algunos casos las variantes causativas en la alternancia lábil resultan forzadas. Pongamos como ejemplo los verbos adelgazar y envejecer: las causas de (21-22, extraidos del CREA) se interpretan como indirectas desde el punto de vista cognitivo, aunque estén codificadas como directas; la causativa puede incluso denotar una propiedad del sujeto -ser adelgazante-, en lugar de un evento, como muestra la anomalía de los ejemplos de (23):

(21) a. Los únicos alimentos que adelgazan son los que se quedan en el plato sin ser consumidos.

b. Aunque soy más bien delgada, mis brazos son muy gordos. ¿Lograría adelgazarlos con algún ejercicio?

c. Imagina una cuerda tendida desde el lavabo hasta la cabecera de su cama y de ella cuelgan todos los gatos de don Angelito, adelgazados por la muerte, hay qué ver cómo adelgaza la muerte a los gatos, y a los conejos, como si fueran animales llenos de fingimiento de tamaño, llenos de aire.

(22) a. [...] como creo haber oído decir a Aranguren, el poder no solo corrompe, sino que también derechiza y envejece. 
b. Tanto dolor produce la jubilación que envejece a los hombres más que la edad.

c. Solo hay que comparar la manera en que la vida contemplativa conserva a los pintores, Tiziano, Picasso, Miró, Dalí, Balthus... con la manera en que la vida de esfuerzo físico envejece a los deportistas.

(23) a. ?Dejar alimentos en el plato ha adelgazado a Pepe.

b. ?Yo adelgacé mis brazos con unos ejercicios específicos.

c. ?La muerte ha adelgazado al gato.

En realidad, estos verbos se emplean más en causativas analíticas que en léxicas (24); además, los adjetivos de los que derivan se combinan frecuentemente con verbos pseudocopulativos, los cuales participan, a su vez, de la alternancia anticausativa -mediante la adición de se-como vemos en (25) con enfermo, del que deriva enfermar con un 7'92\% de usos causativos. Los ejemplos son de CREA:

(24) a. [...] el hecho de contar con un producto que ha hecho adelgazar a los ratones no necesariamente significa que se pueda lograr lo mismo en los humanos.

b. Pero la película [...] respira como entonces, con naturalidad y fluidez, sin baches ni tropiezos, sin una caída de ritmo ni una "modernidad" que la haya hecho envejecer prematuramente.

(25) a. Otras veces yo veo que la gente hasta me quiere poner enfermo, volverme loco, haciéndome esas cosas.

b. El profesorado tiene derecho a ponerse enfermo o a casarse y que la Ley le ampare.

No obstante, el hecho de que ciertos verbos alternen poco o, para algunos hablantes, nunca, no significa que "causativicen" en el sentido de que la variante inacusativa sea la básica frente a verbos como abrir, con los que esa misma variante parecería la derivada mediante un proceso de intransitivización. El problema de la direccionalidad de la alternancia ha recibido mucha atención en la bibliografía desde LyR (1995) y hoy en día existe bastante acuerdo respecto a la idea de que no hay variantes básicas ni derivadas ${ }^{18}$ (cf. Schäfer 2008, Vivanco 2016). Los verbos de cambio de estado en general, salvo los agentivos (asesinar), no especifican si el cambio en cuestión ha de ser desencadenado por una fuerza externa o no, de manera que todos ellos, incluso los del tipo florecer, pueden emplearse para codificar eventos externa o internamente causados, no son básicamente monádicos ni diádicos. Esto no es incompatible con que algunos de ellos se empleen más frecuentemente para uno de los dos fines o, incluso, que se empleen en exclusiva en la variante inacusativa.

En definitiva, ciertos verbos de la clase B pueden estar resistiéndose al uso generalizado de se porque muchos hablantes los consideren no alternantes y otros los consideren alternantes pero más propensos a suceder espontáneamente; sin embargo ¿qué sucede con verbos como aumentar, con un 44'65\% de usos causativos? Como veremos a continuación, es posible que haya otros factores adicionales implicados.

18 Rappaport-Hovav y Levin (2012), aún manteniéndose en una teoría derivacionalista, defienden actualmente que los verbos alternantes no pueden ser en ningún caso básicamente diádicos, sino que son siempre monádicos. 


\section{UNA PECULIARIDAD ADICIONAL DE ALGUNOS VERBOS LÁBILES: LA AUSENCIA DE DIMENSIÓN ESCALAR}

Es un rasgo característico de los verbos de cambio de estado lexicalizar una escala de propiedad (cf. Rappaport-Hovav, 2008). De acuerdo con Kennedy y McNally (2005), dichas escalas se definen por los siguientes tres parámetros: i) tienen una dimensión de medida (la propiedad), ii) constan de un conjunto de grados, y iii) establecen una relación de orden entre los grados.

No obstante, Fleischhauer y Gamerschlag (2014) llaman la atención sobre ciertos verbos de cambio del alemán que no cumplen con los tres parámetros y defienden que estos lexicalizan la escala dejando algunos parámetros infra-especificados.

Así, el verbo verfärben ('cambiar de color') denota una escala que cumple solo dos de los parámetros - conjunto de grados y dimensión (propiedad cromática) - y carece del tercero. En (26), el argumento cambia a un color que, en este caso, se especifica mediante un adjunto, pero no hay una dirección en el cambio: la camisa puede pasar de rojo a rosa, de azul a amarillo, etc. porque los colores no están ordenados jerárquicamente, a diferencia de los grados de temperatura, edad, etc.

(26) Nach dem Waschen hat sich das Hemd blau verfärbt.

(FyG, 2014: 33)

Tras el lavado ha REFL la camisa azul cambiado.de.color

'Después del lavado, la camisa ha cambiado a color azul / se ha vuelto azul'.

El verbo steigen ('elevar', 'aumentar', 'subir') -y otros como zunehmen ('aumentar') y fallen ('caer')-, por su parte, no denota ninguno de los tres parámetros de la escala. En (27) la dimensión y el conjunto de grados vienen dados por el argumento (precio, temperatura), pero no por el verbo: al cambiar el argumento cambia la escala -(a) frente a (b)- ${ }^{19}$ No hay, además, una relación de orden, pues el verbo especifica exclusivamente la dirección (ascendente) del cambio. Lo mismo ocurre exactamente con los verbos aumentar y disminuir del español: en (28a-d) observamos que cada argumento aporta una dimensión de medida diferente -número, caudal, temperatura, pasión-, un conjunto de grados y una relación de orden entre ellos, mientras que el verbo indica tan solo la dirección del cambio.

(27) a. Der Preis des Apertments steigt.

(FyG, 2014: 34)

el precio de.GEN apartamentos aumenta

'El precio de los apartamentos está aumentando'.

b. Der Druck in der Kabine steigt.

La presión en la cabina aumenta

'La presión en la cabina está aumentando'.

(28) a. El número de becarios ha disminuido dramáticamente.

b. El caudal del río Moldava disminuyó el pasado verano.

c. La temperatura de la biblioteca está disminuyendo.

d. Su pasión por la literatura fue disminuyendo con los años.

19 Nótese que el hecho de que el argumento pueda contribuir a la información escalar está relacionado con que ésta se predica de él y ambos forman un constituyente. 
El verbo (sich) ändern ('cambiar'), igual que el verbo cambiar del español (29), solo denota el conjunto de grados - que se limita a dos-, pero no aporta por sí mismo la dimensión de la escala - la cual viene dada, de nuevo, por el argumento- ni la relación de orden entre los grados, o tampoco la dirección del cambio. Nótese que los argumentos de (29c-d) ni siquiera aportan una dimensión de medida o una relación de orden: en (29c) mi vida pasa de tener una propiedad no especificada a tener otra diferente, y en (29d) el clima pasa de ser una manera no especificada a ser de otra. Es decir, este verbo denota un cambio del tipo A $\rightarrow$ B sin especificar qué propiedad define A ni cuál define B.

(29) a. La temperatura ha cambiado.

b. El color de mi pelo está cambiando.

c. Mi vida cambió cuando me mudé.

d. El clima cambió de un día para otro.

Cambiar presenta, de hecho, un comportamiento peculiar en combinación con modificadores del estado resultante: rechaza la lectura restitutiva de otra vez, pero acepta la lectura de duración del resultado de durante si el contexto lo permite:

(30) a. El color de mi pelo cambió otra vez $\rightarrow$ volvió a ser ¿rubio? ¿negro?

b. La temperatura cambió otra vez $\rightarrow$ volvió a ser ¿cálida? ¿fría?

(31) El clima cambió durante una semana.

$\rightarrow$ hacía mucho calor, después refrescó durante una semana

$\rightarrow$ estaba lloviendo, después hizo bueno.

En (30) la razón de que la lectura restitutiva de otra vez no esté disponible no es que no haya un estado resultante, sino que la propiedad de dicho estado no viene definida por el verbo -a diferencia de lo que sucede, por ejemplo, en la temperatura se enfrió otra vez. Lo que ocurre con durante en (31) es que la propiedad del estado resultante puede obtenerse del contexto, y esto permite medir su duración.

La presencia o ausencia de un subevento resultativo en todos los eventos de cambio de estado ha sido tema de debate (véase Schäfer 2008; Labelle y Doron 2010; Cuervo 2003, 2014: Vivanco 2016), también sopensando si esta puede ser una dferencia entre las formaciones lábiles y las anticausativas. El comportamiento anómalo de verbos como cambiar respecto a los diagnósticos que identifican la presencia de un estado resultante puede llevar a confusión; no obstante, la anomalía queda explicada atendiendo a las peculiaridades escalares de este verbo. A nuestro juicio, de acuerdo con lo argumentado en Vivanco (2016) todos los eventos de cambio de estado cuentan, en efecto, con un estado resultate.

Con aumentar y disminuir sucede algo similar: la lectura restitutiva de otra vez y la de duración del estado con durante son posibles, pero no son las más salientes porque no se define el resultado (32-33). La propiedad viene dada por el argumento y, puesto que solo se denota la dirección del cambio, solo sabemos si el resultado consiste en un grado alto o bajo de la propiedad expresada por el SD. Nótese que en la lectura restitutiva de otra vez no es posible determinar, en realidad, si se recupera el estado anterior o no, es decir, no es posible saber si los precios estaban caros antes, o cómo de caros. 
(32) a. Los precios aumentaron otra vez $\rightarrow$ se pusieron caros otra vez.

b. La temperatura aumentó otra vez $\rightarrow$ hizo calor otra vez.

c. El paro aumentó otra vez $\rightarrow$ hubo mucho desempleo otra vez.

(33) a. Los precios aumentaron durante un año $\rightarrow$ estuvieron caros un año

b. La temperatura aumentó durante una semana $\rightarrow$ hizo calor una semana

c. El paro disminuyó durante los meses de verano $\rightarrow$ hubo menos paro en verano

En definitiva, los verbos de cambio de estado que no son propiamente deadjetivales difieren del resto respecto a sus parámetros escalares, que dependen del predicado en su conjunto. Fleischhauer y Gamerschlag (2014) abogan por un análisis unificado de los verbos de cambio de estado que dé cabida a estos casos particulares; para ello, reemplazan la versión fuerte del Principio de Lexicalización de las Escalas de (34) por la versión débil de (35):

(34) Lexicalization of scales (strong version)

(FyG, 2014: 33)

If a change of state verb lexicalizes a scale, all scale parameters are specified in the lexical meaning of the verb.

(35) Lexicalization of scales (weak version)

(FyG, 2014: 33)

A change of state verb lexicalizes a scale, even if one or more of the scale parameters remain unspecified in the meaning of the verb.

Lo importante para el problema que nos ocupa es que aquellos escasos verbos de cambio que no lexicalizan todos los parámetros pertenecen a la clase B en español ${ }^{20}$. Esto ayuda a comprender el comportamiento anómalo que estos verbos exhiben ante ciertos diagnósticos (32-33) y puede ser un factor que contriubuya a su resistencia a la expansión de se como marca regular de la alternancia: se trata de una irregularidad que aleja a estos verbos del patrón estándar de los verbos de cambio de estado $\mathrm{y}$, si bien no es posible afirmar que la ausencia de una dimensión escalar fuerce la ausencia de se, puede considerarse un factor que la desfavorece, pues el clítico guarda una estrecha relación con las propiedades aspectuales y escalares del evento (cf. Vivanco 2016). El estudio pormenorizado de las propiedades aspectuales de los verbos alternantes excede los límites de esta investigación, por lo que remitimos al lector a Folli (2001), Schäfer (2008), Labelle y Doron (2010) y Vivanco (2016).

\section{CONCLUSIONES}

A la luz de la discusión de los apartados anteriores, la resistencia de los veinte verbos lábiles del español a la expansión regular de se está motivada, al menos a nivel sincrónico, por el hecho de que estos reúnen una o varias características especiales.

La mayoría de los verbos de la clase B son de comportamiento aspectual variable por lexicalizar escalas abiertas, un factor determinante en latín que aún opera en romance, y/o tienen grados tan bajos de causalidad que se parecen más a los verbos inacusativos no alternantes que a los alternantes, como envejecer. Otros verbos de comportamiento variable que pertenecen a la clase A - calentar- tienen elevados grados de causalidad, mientras que en el caso de aumentar, que no lleva se pese a su frecuente uso como causativo, interviene

20 No así en alemán, por ejemplo, pues (sich) ändern pertenece a la clase A. 
el factor de la ausencia de dimensión escalar. Otros verbos lábiles, como cambiar, son inherentemente télicos, pero en este caso hemos comprobado que se aúnan el bajo grado de causalidad y la ausencia de dimensión escalar.

Los cambios de estado son prototípicamente télicos - porque dan lugar a un resultado- y prototípicamente escalares - con todos los parámetros de las escalas-, por lo que los verbos lábiles se alejan a estos dos respectos de la norma. Si a ello sumamos su ordenación en una escala de espontaneidad, atendiendo a la hipótesis de la conceptualización y al Principio de la Correspondencia Forma-Frecuencia, obtenemos una imagen compleja pero clara de todo lo que los hace singulares.

Ninguno de los factores mencionados es determinante por sí solo para explicar la ausencia de se, pero aplicados en conjunto hacen que la existencia de la alternancia lábil en español moderno no resulte un fenómeno extraño.

Finalmente, si bien la teoría de la conceptualización incurre en planteamientos circulares cuando deja de utilizarse como herramienta descriptiva y pasa a considerarse una hipótesis explicativa, el uso del Principio de la Correspondencia Forma-Frecuencia contribuye notablemente a su adecuación empírica y teórica, como ha quedado demostrado en este trabajo.

\section{Referencias bibliográficas}

Alexiadou, A. (2014). "The problem with internally caused change of state verbs", Linguistics, 52 (4), 879-909.

Alexiadou, A. y Anagnostopoulou, E. (2004). "Voice morphology in the causative-inchoative alternation: Evidence for a non-unified structural analysis of unaccusatives". En Alexiadou, A., E. Anagnostopoulou y M. Everaert (eds.). The unaccusativity puzzle: Explorations of the syntax-lexicon interface. Oxford: Oxford University Press, pp. 114-136.

Alexiadou, A., Anagnostopoulou, E. y Schäfer, F. (2006). "The properties of anticausatives crosslinguistically”. En M. Frascarelli (ed.). Phases of interpretation. Berlín: Mouton, pp. 187-211.

Alexiadou, A., Anagnostopoulou, E. y Schäfer, F. (2015). External arguments in transitivity alternations. A layering approach. Oxford: Oxford University Press.

Arad, M. (2005). Roots and patterns: Hebrew morpho-syntax. Dordrecht: Springer.

Bosque, I. (1990): "Sobre el aspecto en los adjetivos y participios. En I. Bosque (ed.). Tiempo y aspecto en español. Madrid: Cátedra, pp. 1977-214.

Bybee, J. L. (2006). "From usage to grammar: The mind's response to repetition". Language, 82 (4), pp. 711-733.

Cennamo, M. (2012). “Aspectual constraints on the (anti)causative alternation in Old Italian". En Barddal, J., M. Cennamo y E. Van Gelderen (eds.). Variation and change in argument realization. Thematic issue of transactions of the philological society, 110 (3), pp. 394-421.

Cennamo, M. Barddal, J. y Eythorsson, T. (2015). "Semantic and (morpho-)syntactic constraints on anticausativization: Evidence from Latin and Old Norse-Icelandic”. Linguistics, 53 (4), pp. 677-729.

Cennamo, M. y Jezek, E. (2011). "The anticausative alternation in Italian". En Massariello, G. y S. Dal Masi (eds.). Le interfacce. Roma: Bulzoni, pp. 809-823.

Centineo, G. (1995): “The distribution of $s i$ in Italian transitive / inchoative pairs". En Simons, M. y T. Galloway (eds.). SALT V. Ithaca, N.Y.: Cornell University, pp. 54-71.

Chierchia, G. [1989] (2004). "A semantics for unaccusatives and its syntactic consequences". En Alexiadou, A., E. Anagnostopoulou, y M. Everaert (eds.). The unaccussativity puzzle: Explorations of the syntax-lexicon interface. Oxford: Oxford University Press, pp. 22-59.

Croft, W. (2003). Typology and universals. Cambridge, Mass.: Cambridge University Press. 
Fadlon, J. (2012). "Hidden entries: A psycholinguistic study of derivational gaps". En Everaert, M., M. Marelj y T. Siloni (eds.). The theta system: Argument structure at the interface. Oxford: Oxford University Press, pp. 200-226.

Feltenius, L. (1977). Intransitivizations in Latin. Uppsala: Almqvist \& Wiksell.

Fleishhauer, J. y Gamerschlag, T. (2014). "We are going through changes: How change of state verbs and arguments combine in scale composition". Lingua, 141, pp. 30-47.

Folli, R. (2001). Constructing telicity in English and Italian. Oxford University [tesis doctoral].

Gianollo, C. (2014). "Labile verbs in Late Latin”. Linguistics, 52 (4), pp. 945-1002.

Givón, T. (1991): "Isomorphism in the grammatical code. Cognitive and biological considerations". Studies in Language, 15, pp. 85-114.

Greenberg, J. H. (1966). Language universals, with special reference to feature hierarchies. The Hague: Mouton.

Harley, H. y Noyer, R. (2000). "Formal vs. encyclopedic properties of vocabulary: evidence from nominalization." En Peters, B. (ed.). The Lexicon-Encyclopedia Interface. Amsterdam: Elsevier, pp. 349-74.

Haspelmath, M. (1993): "More on the typology of inchoative / causative alternations". En Comrie, B. y M. Polinsky (eds.). Causatives and transitivity. Amsterdam: John Benjamins, pp. 87-120.

Haspelmath, M. (2008). "Frequency vs. iconicity in explaining grammatical asymmetries". Cognitive Linguistics, 19 (1), pp. 1-33.

Haspelmath, M., Calude, A., Spagnol, M. Narrog, H. y Bamyaci, E. (2014). "Coding causal-noncausal verb alternations: A form-frequency correspondence explanation”. Journal of Linguistics, 50 (3), pp. 587-625.

Heidinger, S. (2014): "The persistence of labile verbs in the French causative-anticausative alternation". Linguistics, 52 (4), pp. 1003-1024.

Heidinger, S. (2015). "Causalness and the encoding of the causative-anticausative alternation in French and Spanish". Journal of Linguistics, 51 (3), pp. 562-594.

Horvath, J. y Siloni, T. (2008). “Active lexicon: Adjectival and verbal passives". En Danon, G., S. Armon-Lotem, y S. Rothstein (eds.). Generative approaches to Hebrew linguistics. Amsterdam: John Benjamins.

Jiménez-Fernández, Ángel y Mercedes Tubino (2014). "Variación sintáctica en la causativización lexica”. RSEL, 44 (1), pp. 7-37.

Kailuweit, R. (2012). "Construcciones anticausativas: El español comparado con el francés”. En Bellosta von Colbe, V. y García García, M. (eds.). Aspectualidad - transitividad - referencialidad. Las lenguas románicas en contraste. Frankfurt: Peter Lang, pp. 133-158.

Karantzola, E. y Lavidas, N. (2014). "On the relation between labilizations and neuter gender: Evidence from Greek diachrony”. Linguistics, 52 (4), pp. 1025-1060.

Kennedy, C. y McNally, L. (2005). "Scale structure, degree modification and the semantics of gradable predicates". Language, 81, pp. 345-381.

Labelle, M. (1992). "Change of state and valency." Journal of Linguistics, 28, pp. 375-414.

Labelle, M. y Doron, E. (2010). "Anticausative derivations (and other valency alternations) in French." Probus, 22 (2), pp. 303-16.

Lavidas, N. (2009). Transitivity alternations in diachrony: Changes in argument structure and voice morphology. Newcastle: Cambridge Scholars Publishing.

Letuchyi, A. (2010). "Lability and spontaneity". En Brand, P. y M. García García (eds.). Transitivity. Form, meaning, aquisition and processing. Amsterdam: John Benjamins, pp. 237-256.

Levin, B. y Rappaport Hovav, M. (1995). Unaccusativity. At the syntax-semantics interface. Cambridge, Mass.: MIT Press.

Malkiel, Y. (1941). “Atristar-entristecerse. Adjectival verbs in Spanish, Portuguese and Catalan". Studies in Philology, Vol. XXXVIII (3), pp. 429-462. 
McKoon, G. y Macfarland, T. (2000). "Externally and internally caused change of state verbs". Language, 76, pp. 833-858.

Nedjalkov, V. P. (1969). "Nekotorye verojatnostnye universalii v glagol'nom slovoobrazovanii". En I. F. Vardul' (ed.). Jazykovye versalii i lingvisticeskaja tipologija. Moscú: Nauka, pp. 106-114.

Nedjalkov, V. P. y Silinsky, G. G. (1973). "The typology of morphological and lexical causatives". En Kiefer, F. (ed.). Trends in soviet theoretical linguistics. Dordrecht: Reidel.

Rappaport Hovav, M. (2014). "Lexical content and context: The causative alternation in English revisited". Lingua, 141, pp. 8-29.

Rappaport Hovav, M. y Levin, B. (2012). "Lexicon uniformity and the causative alternation". En Everaert, M., M. Marelj y T. Siloni (eds.). The theta system: argument structure at the interface. Oxford: Oxford University Press, pp. 150-176.

Real Academia Española: Banco de datos (CREA) [en línea]. Corpus de Referencia del Español Actual. <http://www.rae.es> [03/02/2017]

Reinhart, T. (2000): The theta system: syntactic realization of verbal concepts, OTS Working papers, 00.01/TL, University of Utrecht.

Rothemberg, M. (1974). Les verbes à la fois transitifs et intransitifs en français contemporain. La Haye: Mouton.

Schäfer, F. (2008). The syntax of (anti)causatives. External arguments in change of state contexts. Amsterdam: John Benjamins.

Vivanco, M. (2016). Causatividad y cambio de estado en español. La alternancia causativo-inacusativa. Universidad Complutense de Madrid [tesis doctoral].

Wright, S. (2001). Internally caused and externally caused change of state verbs. Northwestern University [tesis doctoral].

Wright, S. (2002). "Transitivity and change of state verbs". BLS, 28, pp. 339-350.

Zipf, G. K. (1935). The psycho-biology of language. Boston: Houghton Mifflin. 